\title{
Epithelial-mesenchymal Transition is Associated with Acquired Resistance to 5-Fluorocuracil in HT-29 Colon Cancer Cells
}

\author{
A-Young Kim ${ }^{1 \#}$, Jae-Hwan Kwak²", Nam Kyung Je', Yun-hee Lee ${ }^{3}$ and Young-Suk Jung \\ ${ }^{1}$ College of Pharmacy, Pusan National University, Busan, Korea \\ ${ }^{2}$ College of Pharmacy, Kyungsung University, Busan, Korea \\ ${ }^{3}$ College of Pharmacy, Yonsei University, Incheon, Korea
}

(Received June 14, 2015; Revised June 19, 2015; Accepted June 24, 2015)

\begin{abstract}
5-Fluorouracil (5-FU) is commonly used for the therapy of colon cancer; however, acquired resistance to 5-FU is a critical barrier to successful treatment and the primary cause of chemotherapy failure. Epithelialmesenchymal transition (EMT) is a process whereby cells undergo alterations in morphology and molecular characteristics promoting tumor progression and metastasis. Accumulating evidence shows that transition from epithelial to mesenchymal phenotype in cancer cells is associated with their resistance to chemotherapy. However, it is still poorly understood whether EMT is involved in acquired resistance to 5FU. In this study, we developed an in vitro cell model, 5-FU-resistant HT-29 colon cancer cells, and characterized the differences in cellular morphology and molecular alterations between parental and resistant cells. In accord with mesenchymal-like morphology of 5-FU-resistant HT-29 cells, the expression of the mesenchymal marker fibronectin was significantly increased in these cells in comparision with that in the parental cells. Of interest, we also found a marked increase in the expression of EMT-inducing transcription factors Twist, Zeb1, and Zeb2. Finally, 5-FU-resistant cells showed enhanced migration in comparison with parental HT-29. Taken together, these results indicate that EMT could be associated with 5-FU resistance acquired by HT-29 cells. A specific role of each transcription factor found in this study will require further investigation.
\end{abstract}

Key words: 5-Fluorouracil, Acquired resistance, Epithelial-mesenchymal transition, Colon cancer

\section{INTRODUCTION}

Colon cancer has a high incidence and is one of the leading causes of cancer-related death in the elderly (1). As a first-line chemotherapeutic agent for colon cancer, 5-fluorouracil (5-FU) is used to increase the probability of survival (2). The structure of 5-FU is similar to those of pyrimidines in DNA and RNA; 5-FU inhibits thymidylate synthase and is incorporated into RNA and DNA, resulting in cytotoxicity and cell death (3). Although the understanding of the

Correspondence to: Yun-hee Lee, College of Pharmacy, Yonsei University, Incheon 406-840, Korea

E-mail: yunlee.lee@yonsei.ac.kr

Young-Suk Jung, College of Pharmacy, Pusan National University, Busan 609-735, Korea

E-mail: youngjung@pusan.ac.kr

"Kim AY and Kwak JH contributed equally to this work.

This is an Open-Access article distributed under the terms of the Creative Commons Attribution Non-Commercial License (http:// creativecommons.org/licenses/by-nc/3.0) which permits unrestricted non-commercial use, distribution, and reproduction in any medium, provided the original work is properly cited. mechanism of 5-FU action has greatly increased in recent years, the development of drug-resistant phenotypes remains a critical limitation to its clinical use (4). The molecular mechanisms of therapeutic resistance to 5-FU are poorly understood, and investigation of molecular pathways associated with chemo-resistance could lead to novel strategies for the treatment of patients with 5-FU-resistant colon cancer.

Epithelial-mesenchymal transition (EMT) is a multi-step morphogenetic process in which epithelial cells downregulate their epithelial properties and acquire mesenchymal characteristics, leading to remodeling of the cytoskeleton and enhancement of migration potential, which is a functional hallmark of EMT (5). The EMT phenotype is characterized by the loss of cell-to-cell adhesion $(6,7)$. Another hallmark of EMT is the loss of the epithelial adhesion molecule E-cadherin and gain of mesenchymal markers such as vimentin and/or fibronectin. Suppression of E-cadherin expression is mediated by several genetic factors or direct promoter repression mediated by Snail, Slug, Twist, Zeb1, and Zeb2 (6-9), all of which interact with E-box elements located within the proximal region of the E-cadherin promoter (6). Remarkably, the signaling pathway that involves 
these EMT-inducing transcription factors overlaps significantly with each other. Specifically, Snail regulates the expression of Zeb factors and also increases the stability of Twist, followed by activation of transcription of the Slug gene. Thus, it is suggested that EMT-inducing transcription factors cooperate with each other and their specificity might affect spatial and temporal regulation of EMT.

Accumulating evidence suggests that EMT is associated with acquired resistance to cancer therapy as well as cancer metastasis (10). Recently, an EMT phenotype has been identified in a number of cancer cells, including gefitinibresistant non-small cell lung cancer cells (NSCLC) (11), paclitaxel-resistant ovarian cancer cells (12), and tamoxifen-resistant breast cancer cells (13). Furthermore, cancer cells surviving in breast cancer patients after conventional therapy possess mesenchymal characteristics and tumor-initiating features (14). In agreement with these findings, restoration of the epithelial phenotype increases sensitivity of tumor cells to chemotherapy (14). Taken together, these studies suggest that EMT not only increases metastasis, but is also involved in drug resistance in cancer cells.

During the establishment of 5-FU-resistant cells, we found that cells with the 5-FU-resistant phenotype showed morphological changes in comparison with parental HT-29 cells. The present study focused on the alterations in the expression levels of the genes related to EMT.

\section{MATERIALS AND METHODS}

Materials. 5-Fluorouracil (5-FU) and crystal violet were purchased from Sigma Aldrich (St. Louis, MO, USA). Anti-E-cadherin antibodies were obtained from Cell signaling (Danver, MA, USA). Anti-Fibronectin and anti- $\beta$-actin antibodies were purchased from Santa Cruz Biotechnology (Santa Cruz, CA, USA). Peroxidase conjugated antibodies against mouse or rabbit IgG were obtained from Thermo scientific (Waltham, MA, USA).

Cell culture. HT-29 parental cell line was obtained from Korea Research Institute of Bioscience and Biotechnology (KRIBB, Cheongwon, Chungbuk, Korea). HT-29 parental cell line was cultured in McCoy's 5A Medium (Hyclone, Logan, Utah, USA). It was grown in media supplemented with 10\% Fetal Bovine Serum (FBS) and 100 U/
$\mathrm{mL}$ penicillin and $100 \mu \mathrm{g} / \mathrm{mL}$ streptomycin (GenDEPOT, Barker, TX, USA) at $37^{\circ} \mathrm{C}$ in a humidified incubator with $5 \% \mathrm{CO}_{2}$. 5-FU resistant phenotype was established by repeated exposure to 5-FU.

Cell viability assay. Cell viability was assessed by EZCyTox (Daeil Lab Service, Seoul, Korea). $0.5 \times 10^{4}$ cells were plated in 96-well plate. After $24 \mathrm{~h}$, cells were treated with indicated concentrations of 5-FU. EZ-CyTox solution was added to each well and it was measured at a $450 \mathrm{~nm}$. The results were expressed as a percentage compared to the vehicle treated cells.

RNA extraction and semi-quantitative RT-PCR. Total RNA was purified from cells using the NucleoSpin ${ }^{\circledR}$ RNA (MACHEREY-NAGEL, Dueren, Germany). cDNA synthesis was performed with iScript ${ }^{\mathrm{TM}}$ cDNA Synthesis system (Bio-Rad, Hercules, CA, USA), followed by PCR using GoTaq Flexi DNA Polymerase (Promega, Madison, WI, USA). PCR products were analyzed by gel electrophoresis on a $1 \%$ agarose (w/v) gel. The sequences of human specific primers for E-cadherin, fibronectin, Twist, Zeb1, Zeb2, and $18 \mathrm{~S}$ are provided in Table 1.

Western blotting analysis. Cells were lysed with icecold PRO-PREP ${ }^{\mathrm{TM}}$ protein extract solution (iNtRON, Sungnam, Gyunggi, Korea) and protein concentration was quantified using the BCA procedure (Thermo scientific, Waltham, MA, USA). Equal amounts of protein samples were separated by SDS-PAGE using $8 \%$ or $10 \%$ polyacrylamide gel and then transferred onto a polyvinylidene difuoride (PVDF) membrane (Millipore, Billerica, MA, USA). The membrane was blocked with $5 \%$ skim milk in $100 \mathrm{mM}$ Tris-HCl (pH 7.5), $150 \mathrm{mM} \mathrm{NaCl}$, and $0.07 \%$ Tween-20 (TBST) for $1 \mathrm{~h}$ at room temperature. The membranes were incubated with TBST containing 5\% milk and the primary antibodies. After three washes with TBST, the blot was incubated with the appropriate horseradish peroxidase-conjugated secondary antibodies. The antigen was detected using an Western Bright ECL HRP substrate kit (Advansta, Menlo Park, CA, USA).

Migration assay. 24-Transwell migration chamber (Corning Life Sciences, Corning, NY, USA) was used to

Table 1. List of human primers used for RT-PCR

\begin{tabular}{lll}
\hline \hline Primer & Forward & Reverse \\
\hline E-cadherin & 5'-TCCATTTCTTGGTCTACGCC-3' & 5'-CACCTTCAGCCATCCTGTTT-3' \\
Fibronectin & 5'-TCGAGGAGGAAATTCCAATG-3' & 5'-CTCTTCATGACGCTTGTGGA-3' \\
Twist & 5'-GGAGTCCGCAGTCTTACGAG-3' & 5'-TCTGGAGGACCTGGTAGAGG-3' \\
Zeb1 & 5'-GCACAACCAAGTGCAGAAGA-3' & 5'-GAACCATTGGTGGTTGATCC-3' \\
Zeb2 & 5'-CAACTCCGATGAACTGCTGA-3' & 5'-AGCCTGAGAGGAGGATCACA-3' \\
$18 \mathrm{~S}$ & 5'-CAGCCACCCGAGATTGAGCA-3' & 5'-TAGTAGCGACGGGCGGTGTG-3' \\
\hline
\end{tabular}


accomplish cell migration assay. Cells were placed in the upper compartment of the migration chamber and incubated for $24 \mathrm{~h}$ at $37^{\circ} \mathrm{C}$. The migrating cells at the bottom side of the membranes stained with crystal violet.

Statistical analysis. All results, expressed as the mean $\pm \mathrm{SD}$, were analyzed by a two-tailed Student's t-test. The acceptable level of significance was established at $P<0.05$.

\section{RESULTS}

Acquisition of 5-FU resistance is associated with morphological changes. To determine the 5-FU sensitivity in our drug-resistant model, cell viability assay was performed following 5-FU treatment. As we expected, cells with the 5-FU-resistant phenotype had significantly increased viability compared with parental HT-29 cells (Fig. 1A). Phase-contrast microscopy revealed a marked alteration of cellular morphology in 5-FU-resistant HT-29 cells (Fig. 1B). Whereas parental cells maintained epithelial morphology, 5-FU-resistant cells exhibited spindle shape, intercellular spaces, and scattering, suggesting loose cell-cell interaction in comparison with parental HT-29 cells.

Cells with 5-FU-resistant phenotype exhibit EMT-like characteristics in comparison with the phenotype of parental HT-29 cells. The observed morphological changes implied that the 5-FU-resistant cells had transitioned to a mesenchymal phenotype. To determine whether these mor-

(A)

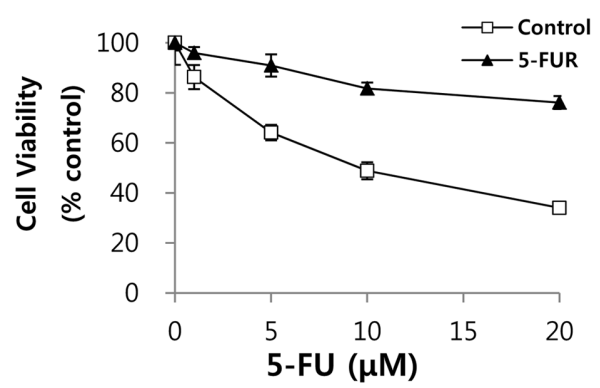

(B)

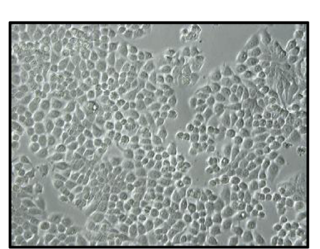

Control

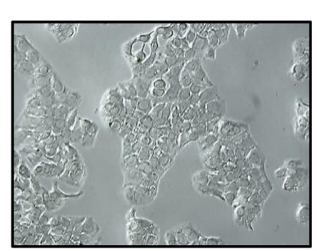

5-FUR
Fig. 1. Acquired $5-\mathrm{FU}$ resistant $\mathrm{HT}-29$ cells show morphological changes compared with parental HT-29 cells. (A) Cell viability was assessed after treatment with increasing concentrations of 5-FU. (B) 5-FU resistant HT-29 cells show significant alteration in cellular morphology (200× magnification).
(A)

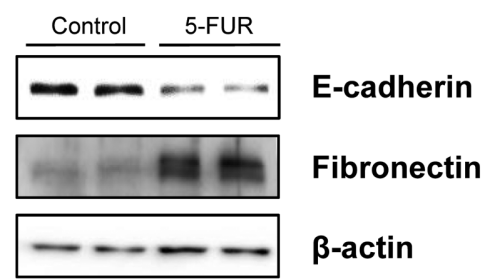

(B)

E-cad

FN
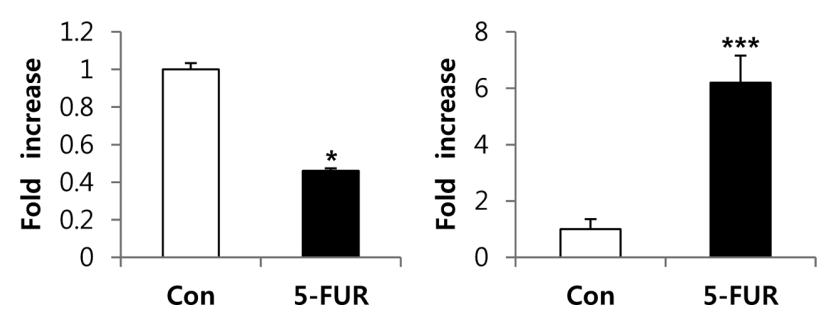

Fig. 2. 5-FU resistant phenotype exhibits an EMT-like cellular characteristics compared with parental HT-29 cells. (A) Protein expression of E-cadherin and fibronection were determined by western blotting. (B) Density of each blot was quantified using Image J. Each value represents the mean \pm SD of three independent experiments. ${ }^{*},{ }^{*}$ Significantly different from the parental HT-29 cells at $p<0.05,0.01$, respectively (Student's $t$-test).

phological changes were associated with EMT, we examined the expression of epithelial and mesenchymal marker proteins. Consistent with morphological changes, 5-FUresistant cells showed down-regulation of the epithelial marker E-cadherin and up-regulation of the mesenchymal marker fibronectin (Fig. 2). These results suggest that EMT could be associated with acquired drug resistance after long-term exposure to 5-FU.

To confirm EMT in 5-FU-resistant cells, we performed RT-PCR analysis of EMT markers and EMT-inducing transcription factors. As shown in Fig. 3A, the level of the Ecadherin transcript was reduced in 5-FU-resistant cells. In contrast, the expression level of fibronectin was dramatically higher in 5-FU-resistant cells than in the parental cells (Fig. 3A). Consistent with the expression of this EMT marker, the expression of the EMT-inducing transcription factors Twist, Zeb1, and Zeb2 was also significantly increased in 5-FU-resistant cells (Fig. 3B).

5-FU-resistant cells show higher motility than parental HT-29 cells in a transwell migration assay. Epithelial cells undergoing EMT lose their adhesive phenotype and concomitantly acquire higher migratory ability (5). We observed that 5-FU-resistant cells were more motile than parental HT-29 cells, as assessed by a transwell migration assay (Fig. 4). These results confirm that acquisition of the 5-FU-resistant phenotype involves EMT, resulting in higher motile capability, which might be critical for tumor progression. 
(A)

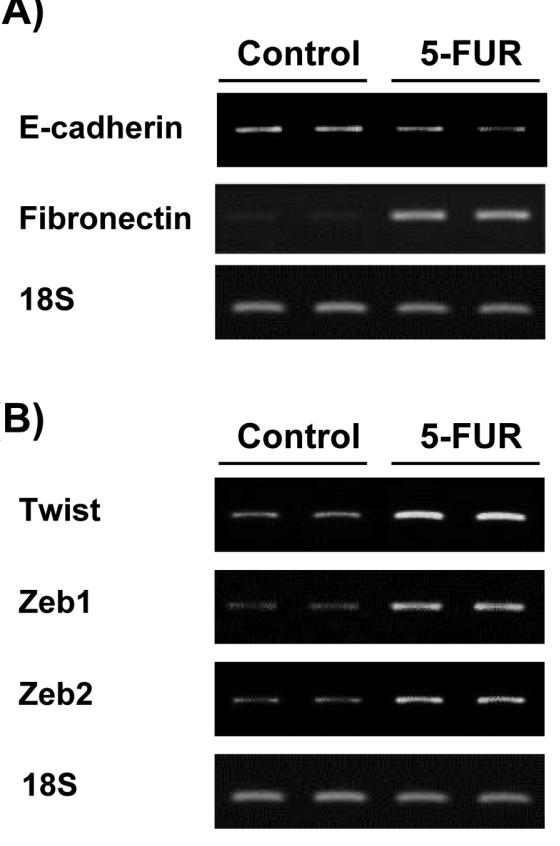

E-cad

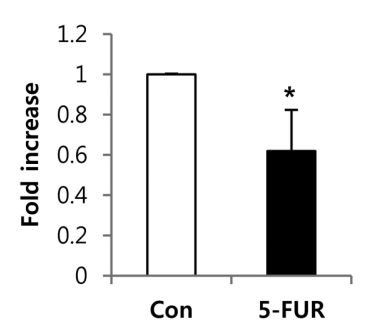

FN

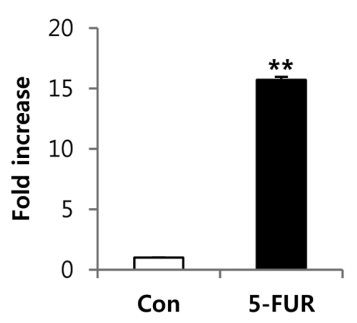

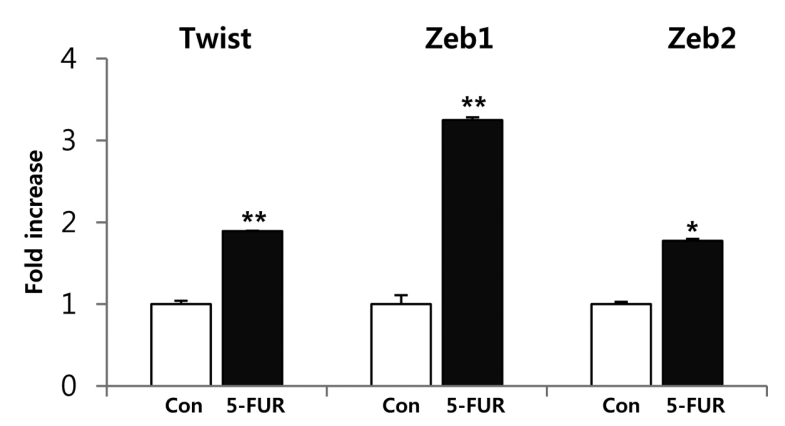

Fig. 3. 5-FU resistant HT-29 cells induce mesenchymal marker accompanied by EMT-inducing transcription factors. RNA expression of (A) EMT markers such as E-cadherin and fibronectin, and (B) EMT-inducing transcription factors Twist, Zeb1, and Zeb2 were analyzed using RT-PCR. Each value represents the mean \pm SD of three independent experiments. * ${ }^{* * *}$ Significantly different from the parental HT-29 cells at $p<0.05,0.001$, respectively (Student's $t$-test).

(A)

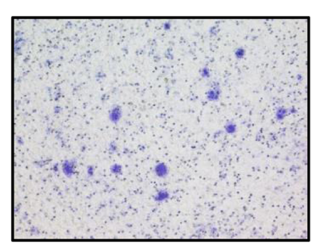

Control

(B)

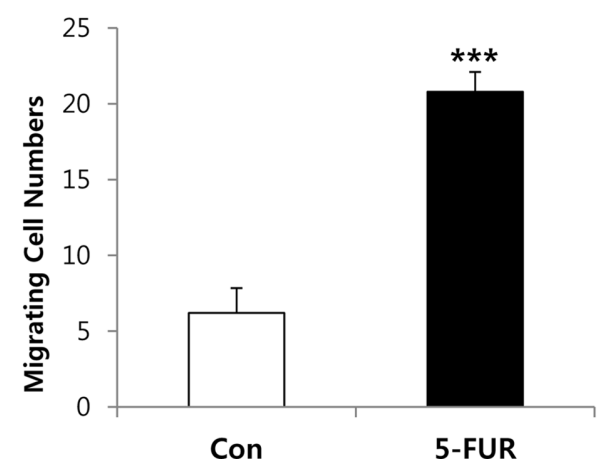

Fig. 4. 5-FU resistant HT-29 cells show higher motility than parental HT-29 cells in transwell migration assay. (A) Cell migration was assessed using 24-transwell migration chamber for $48 \mathrm{hr}$ and cells at the bottom side of the membranes were stained with crystal violet. (B) Migrating cells were counted and plotted as mean \pm SD of three independent experiments. ${ }^{* *}$ Significantly different from the parental HT-29 cells at $p<0.001$ (Student's $t$-test).

\section{DISCUSSION}

5-FU is used frequently in chemotherapy for many kinds of solid tumors. However, patients who initially respond to 5 -FU ultimately become resistant $(15,16)$. In this study, we have established a 5-FU-resistant cell line derived from parental HT-29 colon cancer cells and characterized the phenotype of 5-FU-resistant cells in terms of EMT. Importantly, we found that the expression of the EMT-promoting transcription factors Twist, Zeb1, and Zeb2, which repress E-cadherin transcription, correlates significantly with the 5FU-resistant phenotype in HT-29 colon cancer cells.

Development of drug resistance has been a major barrier to successful treatment and the primary cause of chemotherapy failure. Multiple mechanisms responsible for the drugresistant phenotype in cancer cells have been recognized and recent evidence points to the role of EMT. The link between EMT and chemotherapy resistance has been investigated in NSCLC. Interestingly, the sensitivity of NSCLC to EGFR kinase inhibition depends on their EMT phenotype: mesenchymal-like cells are less sensitive to EGFR inhibition than cells with the epithelial phenotype (17). In line with the letter, the expression of EMT-related genes in lung cancer cell lines is related to their resistance to the inhibitors of both EGFR and PI3K/Akt pathways, which has also been confirmed in a small group of patients (18). Furthermore, in a study that used both surgical specimens 
and cell lines, an EMT-like cellular phenotype was associated with increased resistance to most conventional therapeutic approaches. Of interest, the changes in gene expression during EMT are very similar to the changes in the expression of stem cell markers (19). Notably, residual resistant tumor cells following breast cancer chemotherapy frequently exhibit a stem cell-like phenotype including increased mammosphere formation, which is a characteristic of stem cells (20-22). Recently, the association between EMT-like properties and a stem-cell like phenotype has been reported not only in breast cancer and NSCLC, but also in urinary bladder, head and neck, pancreas, and colorectal carcinoma (23). Thus, the mechanisms of resistance to chemotherapeutic agents might be linked to stem celllike properties of tumor cells that have undergone EMT via the activation of cellular signaling pathways that are common to both processes, such as TGF- $\beta$, Wnt, Notch, and Hedgehog (24).

In summary, cells remaining after long-term exposure to 5-FU showed an EMT phenotype accompanied by acquired drug resistance. Together with other recent studies that suggest the association between EMT and drug resistance, this study might provide a clue for overcoming therapy failure caused by drug resistance. The importance of particular EMT-promoting transcription factors and related signaling pathways in maintaining the EMT phenotype may vary depending on the tissue type (25-28). Therefore, understanding the specific role of each transcription factor found in this study and elucidation of related cellular signaling will require further investigation.

\section{ACKNOWLEDGEMENTS}

This work was supported by a 2-Year Research Grant of Pusan National University.

\section{REFERENCES}

1. Siegel, R., Desantis, C. and Jemal, A. (2014) Colorectal cancer statistics, 2014. Ca Cancer J. Clin., 64, 104-117.

2. Douillard, J.Y., Cunningham, D., Roth, A.D., Navarro, M., James, R.D., Karasek, P., Jandik, P., Iveson, T., Carmichael, J., Alakl, M., Gruia, G., Awad, L. and Rougier, P. (2000) Irinotecan combined with fluorouracil compared with fluorouracil alone as first-line treatment for metastatic colorectal cancer: a multicentre randomised trial. Lancet, 355, 1041-1047.

3. Longley, D.B., Harkin, D.P. and Johnston, P.G. (2003) 5-fluorouracil: mechanisms of action and clinical strategies. Nat. Rev. Cancer, 3, 330-338.

4. Park, I.J., You, Y.N., Agarwal, A., Skibber, J.M., RodriguezBigas, M.A., Eng, C., Feig, B.W., Das, P., Krishnan, S., Crane, C.H., Hu, C.Y. and Chang, G.J. (2012) Neoadjuvant treatment response as an early response indicator for patients with rectal cancer. J. Clin. Oncol., 30, 1770-1776.

5. Thiery, J.P. (2002) Epithelial-mesenchymal transitions in tumour progression. Nat. Rev. Cancer, 2, 442-454.
6. Peinado, H., Olmeda, D. and Cano, A. (2007) Snail, Zeb and bHLH factors in tumour progression: an alliance against the epithelial phenotype? Nat. Rev. Cancer, 7, 415-428.

7. Kang, Y. and Massagué, J. (2004) Epithelial-mesenchymal transitions: twist in development and metastasis. Cell, 118, 277-279.

8. Vandewalle, C., Comijn, J., De Craene, B., Vermassen, P., Bruyneel, E., Andersen, H., Tulchinsky, E., Van Roy, F. and Berx, G. (2005) SIP1/ZEB2 induces EMT by repressing genes of different epithelial cell-cell junctions. Nucleic Acids Res., 33, 6566-6578.

9. Lombaerts, M., van Wezel, T., Philippo, K., Dierssen, J.W., Zimmerman, R.M., Oosting, J., van Eijk, R., Eilers, P.H., van de Water, B., Cornelisse, C.J. and Cleton-Jansen, A.M. (2006) E-cadherin transcriptional downregulation by promoter methylation but not mutation is related to epithelial-to-mesenchymal transition in breast cancer cell lines. Br. J. Cancer, 94, 661-671.

10. Rosanò, L., Cianfrocca, R., Spinella, F., Di Castro, V., Nicotra, M.R., Lucidi, A., Ferrandina, G., Natali, P.G. and Bagnato, A. (2011) Acquisition of chemoresistance and EMT phenotype is linked with activation of the endothelin A receptor pathway in ovarian carcinoma cells. Clin. Cancer Res., 17, 2350-2360.

11. Rho, J.K., Choi, Y.J., Lee, J.K., Ryoo, B.Y., Na, I.I., Yang, S.H., Kim, C.H. and Lee, J.C. (2009) Epithelial to mesenchymal transition derived from repeated exposure to gefitinib determines the sensitivity to EGFR inhibitors in A549, a nonsmall cell lung cancer cell line. Lung Cancer, 63, 219-226.

12. Kajiyama, H., Shibata, K., Terauchi, M., Yamashita, M., Ino, K., Nawa, A. and Kikkawa, F. (2007) Chemoresistance to paclitaxel induces epithelial-mesenchymal transition and enhances metastatic potential for epithelial ovarian carcinoma cells. Int. J. Oncol., 31, 277-283.

13. Hiscox, S., Jiang, W.G., Obermeier, K., Taylor, K., Morgan, L., Burmi, R., Barrow, D. and Nicholson, R.I. (2006) Tamoxifen resistance in MCF7 cells promotes EMT-like behaviour and involves modulation of beta-catenin phosphorylation. Int. J. Cancer, 118, 290-301.

14. Creighton, C.J., Li, X., Landis, M., Dixon, J.M., Neumeister, V.M., Sjolund, A., Rimm, D.L., Wong, H., Rodriguez, A., Herschkowitz, J.I., Fan, C., Zhang, X., He, X., Pavlick, A., Gutierrez, M.C., Renshaw, L., Larionov, A.A., Faratian, D., Hilsenbeck, S.G., Perou, C.M., Lewis, M.T., Rosen, J.M. and Chang, J.C. (2009) Residual breast cancers after conventional therapy display mesenchymal as well as tumor-initiating features. Pro. Natl. Acad. Sci. U.S.A., 106, 13820-13825.

15. Zhang, N., Yin, Y., Xu, S.J. and Chen, W.S. (2008) 5-Fluorouracil: mechanisms of resistance and reversal strategies. Molecules, 13, 1551-1569.

16. Herrmann, R. (1996) 5-Fluorouracil in colorectal cancer, a never ending story. Ann. Oncol., 7, 551-552.

17. Thomson, S., Buck, E., Petti, F., Griffin, G., Brown, E., Ramnarine, N., Iwata, K.K., Gibson, N. and Haley, J.D. (2005) Epithelial to mesenchymal transition is a determinant of sensitivity of non-small-cell lung carcinoma cell lines and xenografts to epidermal growth factor receptor inhibition. Cancer Res., 65, 9455-9462.

18. Byers, L.A., Diao, L., Wang, J., Saintigny, P., Girard, L., Peyton, M., Shen, L., Fan, Y., Giri, U., Tumula, P.K., Nilsson, 
M.B., Gudikote, J., Tran, H., Cardnell, R.J., Bearss, D.J., Warner, S.L., Foulks, J.M., Kanner, S.B., Gandhi, V., Krett, N., Rosen, S.T., Kim, E.S., Herbst, R.S., Blumenschein, G.R., Lee, J.J., Lippman, S.M., Ang, K.K., Mills, G.B., Hong, W.K., Weinstein, J.N., Wistuba, I.I., Coombes, K.R., Minna, J.D. and Heymach, J.V. (2013) An epithelial-mesenchymal transition gene signature predicts resistance to EGFR and PI3K inhibitors and identifies Axl as a therapeutic target for overcoming EGFR inhibitor resistance. Clin. Cancer Res., 19, 279-290.

19. Mani, S.A., Guo, W., Liao, M.J., Eaton, E.N., Ayyanan, A., Zhou, A.Y., Brooks, M., Reinhard, F., Zhang, C.C., Shipitsin, M., Campbell, L.L., Polyak, K., Brisken, C., Yang, J. and Weinberg, R.A. (2008) The epithelial-mesenchymal transition generates cells with properties of stem cells. Cell, 133, 704715 .

20. Shipitsin, M., Campbell, L.L., Argani, P., Weremowicz, S., Bloushtain-Qimron, N., Yao, J., Nikolskaya, T., Serebryiskaya, T., Beroukhim, R., Hu, M., Halushka, M.K., Sukumar, S., Parker, L.M., Anderson, K.S., Harris, L.N., Garber, J.E., Richardson, A.L., Schnitt, S.J., Nikolsky, Y., Gelman, R.S. and Polyak, K. (2007) Molecular definition of breast tumor heterogeneity. Cancer Cell, 11, 259-273.

21. Morel, A.P., Lièvre, M., Thomas, C., Hinkal, G., Ansieau, S. and Puisieux, A. (2008) Generation of breast cancer stem cells through epithelial-mesenchymal transition. PLoS One, 3, e2888.

22. Li, X., Lewis, M.T., Huang, J., Gutierrez, C., Osborne, C.K., Wu, M.F., Hilsenbeck, S.G., Pavlick, A., Zhang, X., Chamness, G.C., Wong, H., Rosen, J. and Chang, J.C. (2008) Intrinsic resistance of tumorigenic breast cancer cells to chemotherapy. J. Natl. Cancer Inst., 100, 672-679.

23. Barr, S., Thomson, S., Buck, E., Russo, S., Petti, F., SujkaKwok, I., Eyzaguirre, A., Rosenfeld-Franklin, M., Gibson, N.W., Miglarese, M., Epstein, D., Iwata, K.K. and Haley, J.D. (2008) Bypassing cellular EGF receptor dependence through epithelial-to-mesenchymal-like transitions. Clin. Exp. Metastasis, 25, 685-693.

24. Singh, A. and Settleman, J. (2010) EMT, cancer stem cells and drug resistance: an emerging axis of evil in the war on cancer. Oncogene, 29, 4741-4751.

25. Elloul, S., Elstrand, M.B., Nesland, J.M., Tropé, C.G., Kvalheim, G., Goldberg, I., Reich, R. and Davidson, B. (2005) Snail, Slug, and Smad-interacting protein 1 as novel parameters of disease aggressiveness in metastatic ovarian and breast carcinoma. Cancer, 103, 1631-1643.

26. Peña, C., García, J.M., Silva, J., García, V., Rodríguez, R., Alonso, I., Millán, I., Salas, C., de Herreros, A.G., Muñoz, A. and Bonilla, F. (2005) E-cadherin and vitamin D receptor regulation by SNAIL and ZEB1 in colon cancer: clinicopathological correlations. Hum. Mol. Genet., 14, 3361-3370.

27. Uchikado, Y., Okumura, H., Ishigami, S., Setoyama, T., Matsumoto, M., Owaki, T., Kita, Y. and Natsugoe, S. (2011) Increased Slug and decreased E-cadherin expression is related to poor prognosis in patients with gastric cancer. Gastric Cancer, 14, 41-49.

28. Yang, J., Mani, S.A., Donaher, J.L., Ramaswamy, S., Itzykson, R.A., Come, C., Savagner, P., Gitelman, I., Richardson, A. and Weinberg, R.A. (2004) Twist, a master regulator of morphogenesis, plays an essential role in tumor metastasis. Cell, 117, 927-939. 